\title{
Singlet night in Feynman-ville: one-loop matching of a real scalar
}

\section{Ulrich Haisch, ${ }^{a}$ Maximilian Ruhdorfer, ${ }^{b}$ Ennio Salvioni, ${ }^{c}$ Elena Venturini ${ }^{b}$ and Andreas Weiler ${ }^{b}$}

${ }^{a}$ Max Planck Institute for Physics, Föhringer Ring 6, 80805 München, Germany

${ }^{b}$ Physik-Department, Technische Universität München, 85748 Garching, Germany

${ }^{c}$ Theoretical Physics Department, CERN, 1211 Geneva 23, Switzerland

E-mail: haisch@mpp.mpg.de, max.ruhdorfer@tum.de, ennio.salvioni@cern.ch, elena.venturini@tum.de, andreas.weiler@tum.de

ABSTRACT: A complete one-loop matching calculation for real singlet scalar extensions of the Standard Model to the Standard Model effective field theory (SMEFT) of dimensionsix operators is presented. We compare our analytic results obtained by using Feynman diagrams to the expressions derived in the literature by a combination of the universal one-loop effective action (UOLEA) approach and Feynman calculus. After identifying contributions that have been overlooked in the existing calculations, we find that the pure diagrammatic approach and the mixed method lead to identical results. We highlight some of the subtleties involved in computing one-loop matching corrections in SMEFT.

Keywords: Beyond Standard Model, Effective Field Theories

ARXIV EPRINT: 2003.05936 


\section{Contents}

1 Motivation $\quad 1$

2 Preliminaries $\quad 3$

3 Calculation $\quad 3$

3.1 Tree-level results $\quad 5$

3.2 One-loop results 5

$\begin{array}{lr}\text { A UOLEA results } & 13\end{array}$

$\begin{array}{ll}\text { B RG evolution of SSM parameters } & 15\end{array}$

\section{Motivation}

The Standard Model (SM) effective field theory aka SMEFT provides a well-defined modelindependent framework to characterise and to constrain new physics that is too heavy to be directly produced in laboratories. This virtue together with the lack of a clear evidence for direct production of new particles at the Large Hadron Collider (LHC) has prompted considerable theoretical and experimental activities that led to the development of the SMEFT framework and its consistent and systematic application to LHC data.

Important steps in the theoretical development of SMEFT were the classification of all independent dimension-six SMEFT operators in [1] (see also [2] for related earlier work) and the calculation of the full one-loop anomalous dimension matrix of these operators in a series of papers [3-6] - partial one-loop and two-loop results have also been obtained in [7-13]. Considerable progress has also been made recently in improving the precision of matching calculations in perturbative extensions of the SM. A complete tree-level dictionary that allows to read off the Wilson coefficients of the dimension-six SMEFT operators in any ultraviolet (UV) completion with general scalar, spinor and vector field content and arbitrary interactions has been presented in [14]. The computation of one-loop matching contributions has been advanced as well by the development of the so-called universal one-loop effective action (UOLEA) approach [15] that generalised methods based on a covariant derivative expansion (CDE) [16] (cf. also [17-19] for earlier works on functional techniques). In its initial formulation the UOLEA did not allow to compute the quantum effects associated to loops involving both heavy and light particles [20] (see also [21]). This shortfall triggered several theoretical improvements aimed at capturing contributions of this type [22-27]. Despite the latter efforts the UOLEA formalism still remains incomplete to date, because a master formula that allows to calculate heavy-light contributions with open derivatives and mixed statistics has so far not been derived in the literature. However, see [28] for recent progress in this direction. 
Computations in the UOLEA framework of the complete set of Wilson coefficients arising at the one-loop level in SMEFT therefore have to make use, at least partially, of conventional Feynman diagram techniques. The article [29] for example has employed the UOLEA master formulae of [26] in combination with Feynman calculus to obtain the first complete one-loop matching corrections at dimension-six for real singlet scalar extensions of the SM (SSM). In this work, we repeat the calculation of [29] from scratch, relying entirely on the use of Feynman diagrams. With the help of our independent computation we are able to identify terms that have been missed in the existing calculations $[26,29]$ - small discrepancies in the latter publications have already been noted in the presentation [30], but updated results have not been published so far. We stress that these discrepancies are due to oversights, and not due to structural limitations of the UOLEA approach. See appendix A for further explanations.

The results presented in this article constitute an important stepping stone to our forthcoming study [31] of the indirect collider sensitivity to pseudo Nambu-Goldstone boson (pNGB) dark matter (DM). This type of DM candidate is characterised by a parametric suppression of its scattering rate on ordinary matter, making it naturally compatible with the null results of direct DM searches. Strong theoretical motivations for this scenario come from composite Higgs models where the Higgs and DM both emerge as pNGBs [32, 33], but the same DM phenomenology can also be realised in simple scalar extensions of the SM see e.g. $[34,35]$. The collider reach on pNGB DM through tree-level production in vector boson fusion has been recently analysed [36], finding a limited sensitivity even at future accelerators. This prompts us to explore one-loop probes, such as for instance off-shell Higgs production, employing an effective field theory (EFT) for the SM plus the DM candidate [31]. A non-trivial aspect of such an analysis is that the virtual DM effects arise at the same order as those of DM-less, one-loop effective operators induced by heavy new physics. To clearly understand the role of these DM-independent effects, it is useful to make use of an explicit model such as that of [35], integrating out the scalar radial mode to obtain precisely the dimension-six SMEFT operators considered in this work. This case study is especially useful because by varying the strength of the parameters, one can effectively interpolate between elementary Higgs-like and strongly-interacting light Higgslike [37] EFT power countings within a simple setup. Given its relevance to our upcoming work [31] and in view of the arguments presented in the previous paragraph, we believe it is worthwhile to provide the complete one-loop matching corrections for the SSM in this short note.

This paper is organised as follows. In section 2 we specify our notation and conventions, while section 3 contains the analytic results of the tree-level and one-loop matching calculation for the SSM. In appendix A we show that the analytic results for the heavy one-loop matching corrections presented in section 3.2 can also be obtained in the UOLEA framework, while in appendix B we collect the anomalous dimensions that describe the renormalisation group (RG) evolution of the SSM parameters and discuss their impact on our one-loop calculations. 


\section{Preliminaries}

In order to set up our notation and conventions, let us first define the electroweak (EW) part of the SM. Before spontaneous EW symmetry breaking the tree-level EW SM Lagrangian takes the following familiar form,

$$
\begin{aligned}
\mathcal{L}_{\mathrm{SM}}= & \left(D_{\mu} H\right)^{\dagger}\left(D^{\mu} H\right)+\mu_{h}^{2}|H|^{2}-\frac{1}{2} \lambda_{h}|H|^{4}-\frac{1}{4} B_{\mu \nu} B^{\mu \nu}-\frac{1}{4} W_{\mu \nu}^{a} W^{a \mu \nu} \\
& +\sum_{f=q, u, d, \ell, e} \bar{f} i \not D f-\left(y_{u} \bar{q} \widetilde{H} u+y_{d} \bar{q} H d+y_{e} \bar{\ell} H e+\text { h.c. }\right)
\end{aligned}
$$

Here $H$ denotes the SM Higgs doublet and the shorthand notation $\widetilde{H}_{i}=\epsilon_{i j}\left(H_{j}\right)^{*}$ with $\epsilon_{i j}$ totally antisymmetric and $\epsilon_{12}=1$ has been used. The covariant derivative is defined as

$$
D_{\mu}=\partial_{\mu}-i g_{1} Y B_{\mu}-i g_{2} \frac{\sigma^{a}}{2} W_{\mu}^{a},
$$

with $g_{1}$ and $g_{2}$ the $\mathrm{U}(1)_{Y}$ and $\mathrm{SU}(2)_{L}$ gauge coupling, respectively, and $B_{\mu}$ and $W_{\mu}^{a}\left(B_{\mu \nu}\right.$ and $W_{\mu \nu}^{a}$ ) the corresponding gauge fields (field strength tensors). The hypercharge operator is denoted by $Y$ with eigenvalues $\left\{Y_{H}, Y_{q}, Y_{u}, Y_{d}, Y_{\ell}, Y_{e}\right\}=\{1 / 2,1 / 6,2 / 3,-1 / 3,-1 / 2,-1\}$ and $\sigma^{a}$ are the Pauli matrices. The Yukawa couplings $y_{u}, y_{d}$ and $y_{e}$ are matrices in flavour space and a sum over flavour indices is implicit in (2.1). Finally, the symbols $q$ and $\ell$ denote left-handed quark and lepton doublets, while $u, d$ and $e$ are right-handed fermion singlets.

As stated before, the goal of this article is to calculate the complete matching corrections up to one-loop order that arise in the SSM. At the renormalisable level, Lorentz and gauge invariance allow a real singlet scalar to couple to the SM exclusively through $|H|^{2}$, and as a result the Lagrangian relevant for the further discussion can be written as

$$
\mathcal{L}_{\phi}=\frac{1}{2}\left(\partial_{\mu} \phi\right)^{2}-\frac{1}{2} M^{2} \phi^{2}-A|H|^{2} \phi-\frac{1}{2} \kappa|H|^{2} \phi^{2}-\frac{1}{3 !} \mu \phi^{3}-\frac{1}{4 !} \lambda_{\phi} \phi^{4} .
$$

Here we have ignored a potential tadpole contribution, meaning that the field $\phi$ in (2.3) corresponds to the excitation around a possible non-zero vacuum expectation value. The parameters $M^{2}, A, \kappa, \mu$ and $\lambda_{\phi}$ appearing in (2.3) are treated as independent in what follows.

\section{Calculation}

By integrating out the field $\phi$ that appears in the SSM Lagrangian

$$
\mathcal{L}_{\mathrm{SSM}}=\mathcal{L}_{\mathrm{SM}}+\mathcal{L}_{\phi},
$$

one can determine the Wilson coefficients $C_{k}$ that multiply the operators $Q_{k}$ in SMEFT

$$
\mathcal{L}_{\mathrm{SMEFT}}=\sum_{k} C_{k} Q_{k},
$$

order by order in perturbation theory by performing a loop expansion

$$
C_{k}=C_{k}^{(0)}+\frac{C_{k}^{(1)}}{(4 \pi)^{2}}+\ldots
$$


where $C_{k}^{(0)}$ and $C_{k}^{(1)}$ denote the tree-level and one-loop coefficients, respectively. The notation introduced in (3.3) will also be used when expanding other quantities of interest. The full set of dimension-six SMEFT operators has been presented in the so-called Warsaw basis in [1]. Up to the one-loop level, it turns out that matching the theory described by the Lagrangian (3.1) to the SMEFT Lagrangian (3.2) generates non-zero Wilson coefficients for the following set of 17 effective operators:

$$
\begin{aligned}
& Q_{H \square}=|H|^{2} \square|H|^{2}, \quad Q_{H u}=\left(H^{\dagger} i \stackrel{\leftrightarrow}{D}_{\mu} H\right)\left(\bar{u} \gamma^{\mu} u\right), \\
& Q_{H}=|H|^{6}, \quad Q_{H d}=\left(H^{\dagger} i \stackrel{\leftrightarrow}{D}_{\mu} H\right)\left(\bar{d} \gamma^{\mu} d\right), \\
& Q_{H D}=\left(H^{\dagger} D_{\mu} H\right)^{*}\left(H^{\dagger} D^{\mu} H\right), \quad Q_{H e}=\left(H^{\dagger} i \stackrel{\leftrightarrow}{D}_{\mu} H\right)\left(\bar{e} \gamma^{\mu} e\right) \\
& Q_{H B}=|H|^{2} B_{\mu \nu} B^{\mu \nu}, \quad Q_{H q}^{(1)}=\left(H^{\dagger} i \stackrel{\leftrightarrow}{D}_{\mu} H\right)\left(\bar{q} \gamma^{\mu} q\right), \\
& Q_{H W}=|H|^{2} W_{\mu \nu}^{a} W^{a \mu \nu}, \quad Q_{H q}^{(3)}=\left(H^{\dagger} i \stackrel{\leftrightarrow}{D_{\mu}^{a}} H\right)\left(\bar{q} \gamma^{\mu} \sigma^{a} q\right), \\
& Q_{H W B}=\left(H^{\dagger} \sigma^{a} H\right) W_{\mu \nu}^{a} B^{\mu \nu}, \quad Q_{H \ell}^{(1)}=\left(H^{\dagger} i \stackrel{\leftrightarrow}{D_{\mu} H}\right)\left(\bar{\ell} \gamma^{\mu} \ell\right), \\
& Q_{u H}=|H|^{2}(\bar{q} \widetilde{H} u), \quad Q_{H \ell}^{(3)}=\left(H^{\dagger} i \stackrel{\leftrightarrow}{D_{\mu}^{a} H} H\left(\bar{\ell} \gamma^{\mu} \sigma^{a} \ell\right),\right. \\
& Q_{d H}=|H|^{2}(\bar{q} H d), \quad Q_{2 y}=\left|\bar{q}_{j} y_{u} u \epsilon^{j i}+\bar{d} y_{d}^{\dagger} q^{i}+\bar{e} y_{e}^{\dagger} \ell^{i}\right|^{2}, \\
& Q_{e H}=|H|^{2}(\bar{\ell} H e) \text {. }
\end{aligned}
$$

Here $\square=\partial_{\mu} \partial^{\mu}, H^{\dagger} i \stackrel{\leftrightarrow}{D}_{\mu} H=i H^{\dagger}\left(D_{\mu}-\overleftarrow{D}_{\mu}\right) H$ and $H^{\dagger} i \stackrel{\leftrightarrow}{D}_{\mu}^{a} H=i H^{\dagger}\left(\sigma^{a} D_{\mu}-\overleftarrow{D}_{\mu} \sigma^{a}\right) H$ and for the operators $Q_{\psi H}$ with $\psi=u, d$, e the sum of the hermitian conjugate in (3.2) is understood.

The matching of (3.1) onto (3.2) can be performed using either Feynman diagrams or functional methods. In fact, the work [26] employed the UOLEA approach to calculate the heavy (i.e. only $\phi$ loops) and the heavy-light (i.e. loops with both $\phi$ and Higgs exchange) one-loop matching corrections for the Wilson coefficients $C_{H \square}$ and $C_{H}$. Based on the results of that article, the paper [29] then presented the complete one-loop matching corrections in the model described by (3.1), computing the missing heavy-light contributions involving a $\phi$ scalar and a gauge boson or a fermion, by means of traditional Feynman diagram techniques.

In contrast to $[26,29]$ our calculation of the Wilson coefficients $C_{k}^{(0)}$ and $C_{k}^{(1)}$ relies on Feynman diagrams only, and therefore represents an independent cross-check of the results obtained earlier. To allow for a direct comparison with the expressions given in the publications [26, 29], we regularise UV divergences using dimensional regularisation (DR) in $d=4-2 \epsilon$ dimensions and renormalise the results in the $\overline{\mathrm{MS}}$ scheme supplemented by the renormalisation scale $\mu_{R}$. Infrared (IR) divergences have also been regularised dimensionally. The matching corrections can therefore be found by simply Taylor expanding the corresponding scattering amplitudes in powers of external momenta squared divided by $M^{2}$ before performing any loop integration. On the other hand, SMEFT loop graphs do not contribute to the matching, because after Taylor expansion of the integrands they involve only scaleless integrals which vanish in DR - see e.g. $[38,39]$ for further technical 

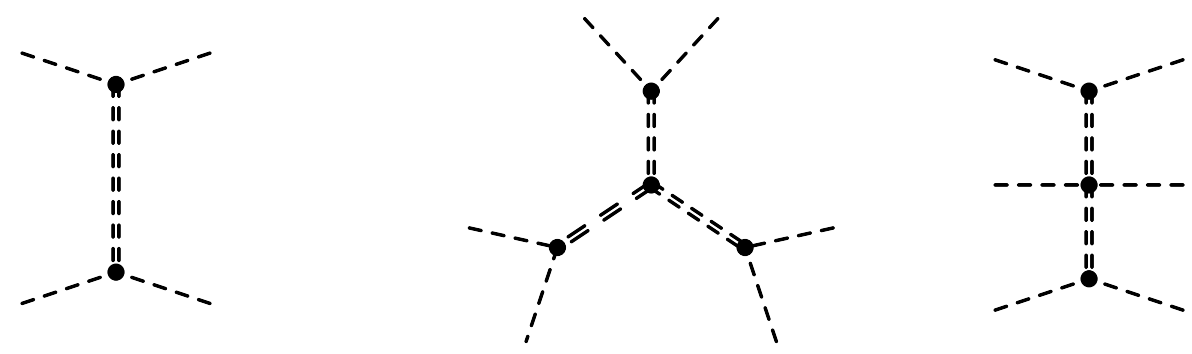

Figure 1. Example diagrams that contribute to the tree-level matching coefficients $\lambda_{h}^{(0)}$ and $C_{H \square}^{(0)}$ (left) and $C_{H}^{(0)}$ (right), respectively. Double-dashed lines represent virtual exchange of the heavy real singlet scalar field $\phi$, while single-dashed lines stand for $H$ or $H^{\dagger}$ fields.

details. The actual generation and computation of the off-shell amplitudes made use of the Mathematica packages FeynArts [40], FeynRules [41], FormCalc [42] and Package-X [43], and part of the one-loop matching corrections obtained by computer were also verified with pen and paper.

\subsection{Tree-level results}

In the SM extension (3.1) only the Higgs quartic $|H|^{4}$ (cf. (2.1)) and the two effective operators $Q_{H \square}$ and $Q_{H}$ (see (3.4)) receive a non-zero matching correction at tree level. The corresponding Feynman diagrams are shown in figure 1. For the additive shift $\lambda_{h}^{(0)}$ of the quartic Higgs coupling, i.e. $\lambda_{h} \rightarrow \lambda=\lambda_{h}+\lambda_{h}^{(0)}$, in agreement with [29] we find

$$
\lambda_{h}^{(0)}=-\frac{A^{2}}{M^{2}},
$$

while in the case of the Wilson coefficients we obtain

$$
\begin{aligned}
C_{H \square}^{(0)} & =-\frac{A^{2}}{2 M^{4}}, \\
C_{H}^{(0)} & =\frac{A^{3} \mu}{6 M^{6}}-\frac{A^{2} \kappa}{2 M^{4}} .
\end{aligned}
$$

The results (3.6) and (3.7) are well-known and agree with the analytic expressions reported for instance in the works $[14,16,29]$.

\subsection{One-loop results}

In order to determine the one-loop matching corrections $C_{k}^{(1)}$ to the Wilson coefficients of the dimension-six SMEFT operators $Q_{k}$ as given in (3.4), we consider only Feynman diagrams that are one-particle-irreducible in the light fields, i.e. we work in the so-called Green's basis defined in [29], subsequently projecting our off-shell results onto the Warsaw basis using the operator identities given in appendix A of the latter paper.

The one-loop matching corrections of the tree-level operators $Q_{H \square}$ and $Q_{H}$ receive contributions from three sources that we describe in the following. The first two types encode the threshold effects at a matching scale $\mu_{M}$ around $M$. The first kind of threshold 

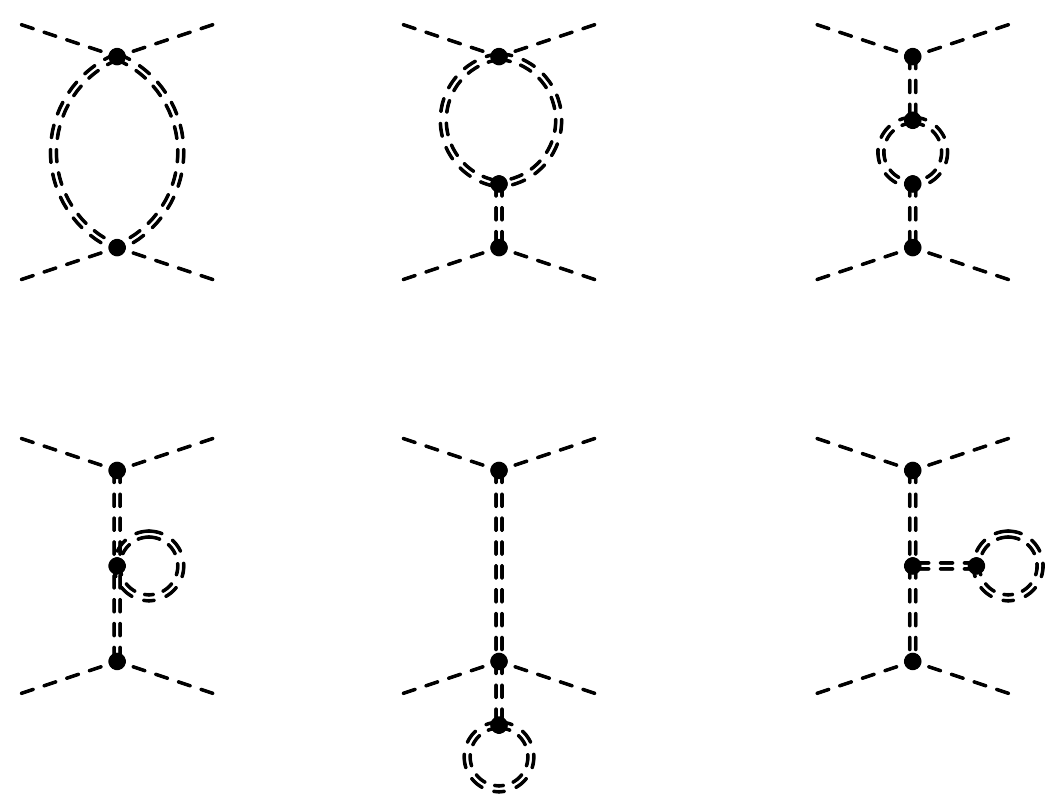

Figure 2. Examples of heavy contributions to the one-loop matching correction $C_{H \square}^{(1)}$. The line styles and their meanings resemble those of figure 1.

corrections arise from heavy loops. For the case of $C_{H \square}^{(1)}$, the relevant graphs are shown in figure 2. Notice that diagrams with $\phi$ tadpoles in general contribute to this type of corrections, hence the analytic expressions for the heavy contributions to the Wilson coefficients $C_{H \square}^{(1)}$ and $C_{H}^{(1)}$ depend on how the tadpole contributions are fixed. In our diagrammatic calculation, as well as in the UOLEA approach described in appendix A, we renormalise $\phi$ tadpoles minimally $[44,45]$ and (3.9), (3.11), (A.8) and (A.9) therefore correspond to the $\overline{\mathrm{MS}}$ scheme. Notice that in the $\overline{\mathrm{MS}}$ scheme the effective one-loop scalar potential contains a term linear in the $\phi$ field, which by definition would be absent in the on-shell scheme where the tadpole counterterm is fixed such that all tadpole diagrams vanish [46] — see also [47-49] for excellent discussions of the different treatments of tadpoles.

The second type of threshold corrections to $C_{H \square}^{(1)}$ and $C_{H}^{(1)}$ stem from heavy-light loop diagrams involving either a $H$ or a $B_{\mu}\left(W_{\mu}^{a}\right)$ field. Universal effects related to the wave function renormalisation of the Higgs field belong to this class. In fact, after the field redefinition $H \rightarrow\left(1-Z_{H}^{(1)} /(4 \pi)^{2}\right) H$ the Wilson coefficients $C_{H \square}$ and $C_{H}$ receive a oneloop contribution proportional to $C_{H \square}^{(0)}$ and $C_{H}^{(0)}$, respectively. The relevant wave function renormalisation constant $Z_{H}^{(1)}$ is determined by calculating the one-loop corrections to the Higgs kinetic term $\left(D_{\mu} H\right)^{\dagger}\left(D^{\mu} H\right)$ that arises from the graph displayed on the left in figure 3. In agreement with [29] we obtain

$$
Z_{H}^{(1)}=\frac{A^{2}}{4 M^{2}}
$$

In addition to the wave function renormalisation contributions, non-universal heavy-light corrections arise. The corresponding scalar and gauge-boson contributions to $C_{H \square}^{(1)}$ are 

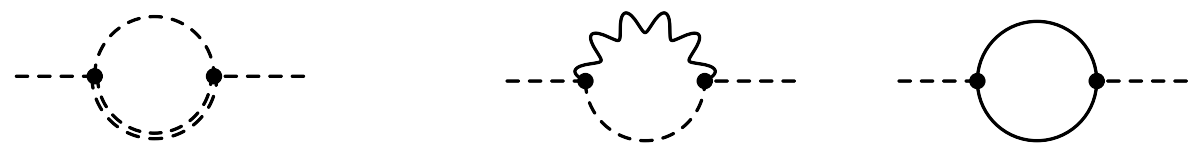

Figure 3. Higgs wave function renormalisation effects. Left: heavy-light contribution to $Z_{H}^{(1)}$ in (3.8). Right: gauge-boson and fermionic contributions to the anomalous dimensions of the SSM parameters $A$ and $\kappa$ in (B.3) and (B.4). The wiggly line corresponds to a $B_{\mu}$ or $W_{\mu}^{a}$ field, the solid straight lines represent fermion fields, while the rest of the line styles and their meanings are identical to those employed in figure 1.
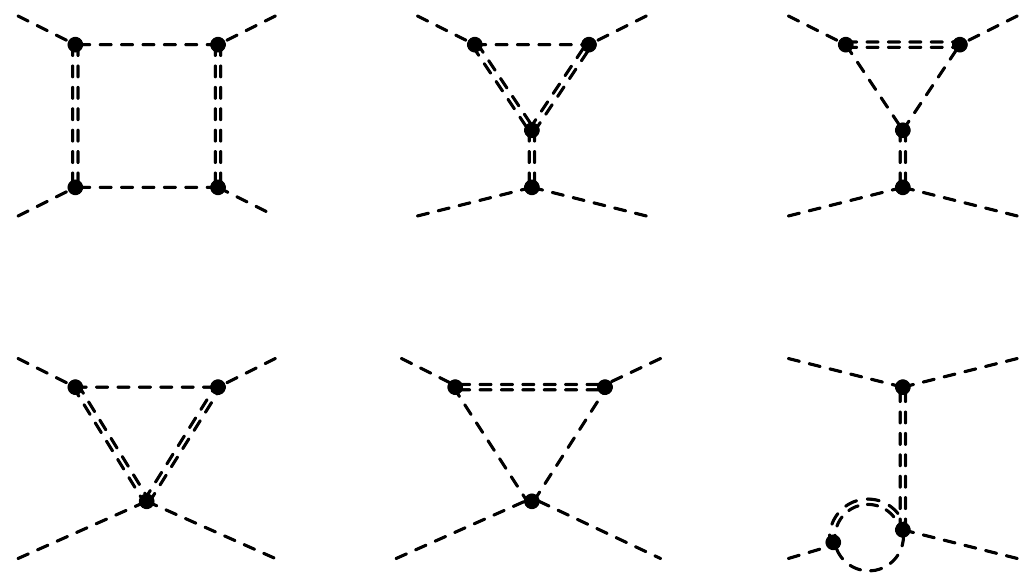

Figure 4. Examples of heavy-light scalar contributions to the one-loop matching correction $C_{H \square}^{(1)}$. Diagrams with loops that contain only $H$ fields are not shown, since they evaluate to zero in DR if the $H$ fields are taken to be massless before Taylor expanding the corresponding loop integrals. The line styles and their meanings resemble those of figure 1.

displayed in figure 4 and figure 5, respectively. Notice that when IR divergences are regulated dimensionally, SSM diagrams involving only light particles in the loop do not need to be considered, because such graphs result in scaleless integrals after Taylor expanding the associated off-shell amplitudes in powers of external momenta squared divided by $M^{2}$. This should be contrasted to methods that use small external momenta or small light-field masses as IR regulators (cf. for instance [50-52]). In these cases, SSM diagrams with only light particles in the loop give non-zero IR divergent corrections but their contributions are exactly cancelled by the corresponding SMEFT graphs. As a result, the one-loop matching corrections $C_{H \square}^{(1)}$ and $C_{H}^{(1)}$ turn out to be independent of the procedure that is used to regulate IR divergences (as they should), and in our calculation we have employed DR to regulate both UV and IR divergences simply because it is technically the easiest method to implement.

The third type of corrections to $C_{H \square}^{(1)}$ and $C_{H}^{(1)}$ arise instead from the renormalisation of the SSM parameters that enter the tree-level Wilson coefficients (see e.g. [53] for a pedagogical discussion). These contributions are, therefore, purely logarithmic in the $\overline{\mathrm{MS}}$ scheme. The logarithmic terms proportional to the SM couplings generate a RG flow that 


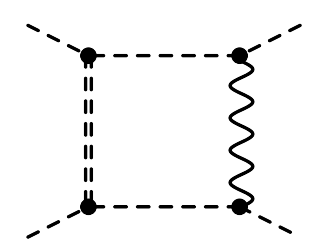

Figure 5. Example of a heavy-light gauge-boson contribution to the one-loop matching correction $C_{H \square}^{(1)}$. Graphs with loops of only $H$ and $B_{\mu}$, or $H$ and $W_{\mu}^{a}$ fields are not displayed, because such diagrams do not contribute if IR divergences are regulated dimensionally. The line styles and their meanings mirror those in figure 3 .
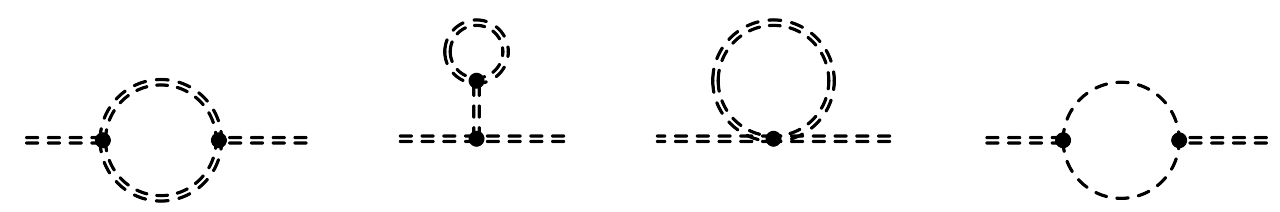

Figure 6. One-loop contributions to the propagator of the real singlet scalar. In the $\overline{\mathrm{MS}}$ scheme the UV poles of the first and second diagram cancel against each other. The line styles and their meanings are identical to those employed in figure 1.

extends below the matching scale, providing one of the contributions to the anomalous dimensions of the SMEFT Wilson coefficients. These effects, coming from light loops, give the same corrections to both the SSM and SMEFT amplitudes. On the other hand, the RG-flow contributions proportional to UV SSM parameters appear only above $\mu_{M}$. The anomalous dimension of $M^{2}$ is obtained from the Feynman diagrams shown in figure 6 , while figure 7 , figure 8 and figure 9 display example graphs of contributions to the running of $A, \kappa$ and $\mu$, respectively. Notice that the anomalous dimensions that describe the RG flow of the SSM parameters $A$ and $\kappa$ also contain pieces arising from the light contributions to the Higgs wave function displayed on the right-hand side in figure 3 , since the corresponding operators contain two powers of the $H$ field. See appendix B for further details and explanations.

In the case of the dimension-six SMEFT operator $Q_{H \square}$, we find after combining the three different types of contributions described above the following result for the one-loop matching correction:

$$
\begin{aligned}
C_{H \square}^{(1)}= & -\frac{\kappa^{2}}{24 M^{2}}+\frac{25 A^{2} \kappa-6 A^{2} \lambda_{\phi}-5 A \kappa \mu}{12 M^{4}}+\frac{38 A^{4}-26 A^{3} \mu+11 A^{2} \mu^{2}}{24 M^{6}} \\
& -\frac{31 A^{2}\left(g_{1}^{2}+3 g_{2}^{2}\right)}{72 M^{4}}+\gamma_{H \square, H \square} C_{H \square}^{(0)} \ln \frac{\mu_{M}}{M} .
\end{aligned}
$$

Here

$$
\gamma_{H \square, H \square}=12 \lambda-\frac{4}{3}\left(g_{1}^{2}+3 g_{2}^{2}\right)+4 y_{2},
$$

with $\lambda$ denoting the quartic Higgs coupling that includes the tree-level shift (3.5) and the objects $C_{H \square}^{(0)}$ and $y_{2}$ defined in (3.6) and (B.8), respectively. Notice that all mass 

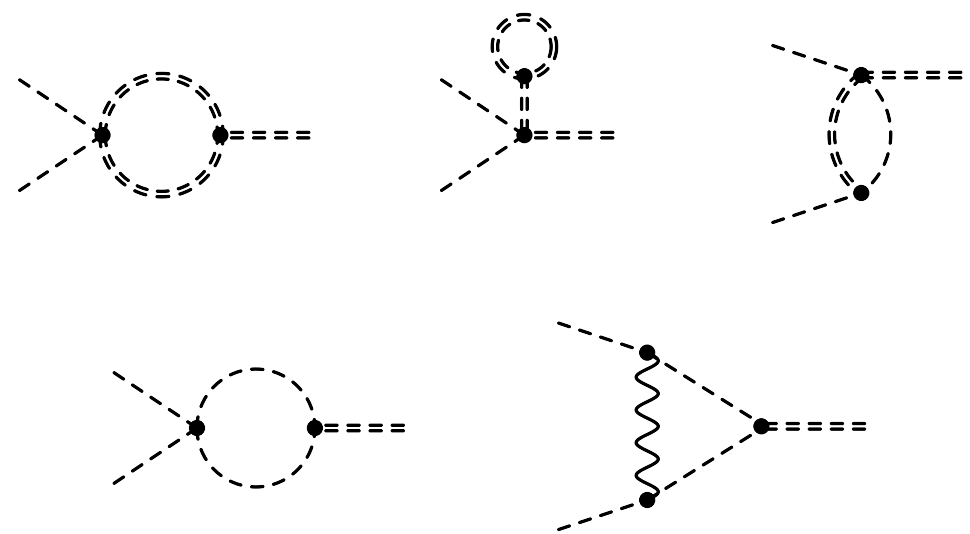

Figure 7. Examples of one-loop contributions to the renormalisation of the coupling $A$. The UV poles of the first and second graph cancel against each other in the $\overline{\mathrm{MS}}$ scheme. The line styles and their meanings are analogue to those of figure 5 .
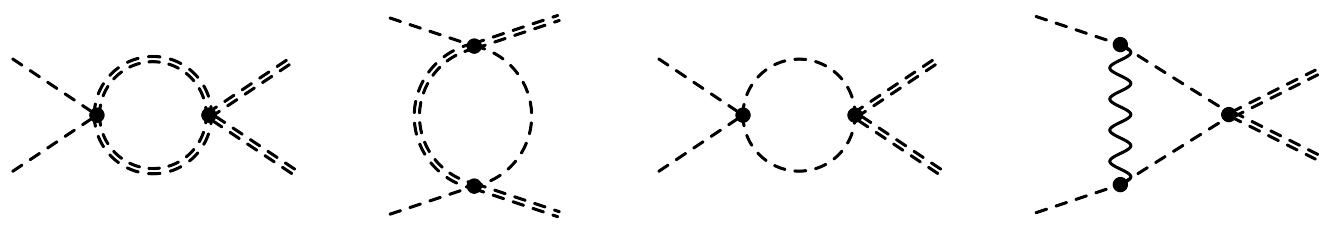

Figure 8. Examples of one-loop contributions to the renormalisation of the coupling $\kappa$. The line styles and their meanings are analogue to those of figure 5 .
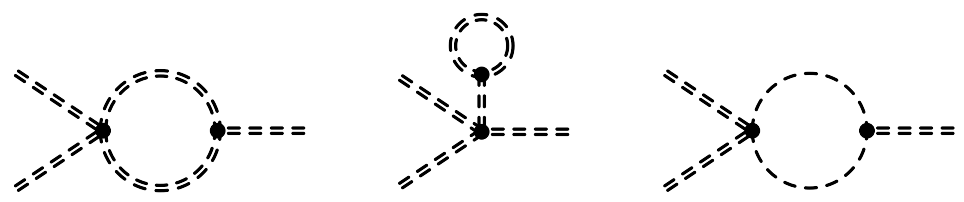

Figure 9. Examples of one-loop contributions to the renormalisation of the coupling $\mu$. The line styles and their meanings resemble those of figure 1 .

and coupling parameters in (3.9), (3.10) as well as in the tree-level expression (3.6) are renormalised at the scale $M$.

A couple of comments concerning our result (3.9) seem to be in order. The rational terms in (3.9) receive contributions from the Higgs wave function renormalisation constant (3.8) and heavy and heavy-light diagrams (see figure 2, figure 4 and figure 5), while the logarithmic terms result from the combination of heavy and heavy-light graphs as well as the renormalisation of $M^{2}$ and $A$ according to (B.2) and (B.3). In fact, the logarithmic pieces proportional to SM couplings combine to give the anomalous dimension $\gamma_{H \square, H \square \text {, }}$ whereas the remaining terms cancel, because they do not run below the matching scale (see appendix B for further details). The anomalous dimension describes the self-mixing of the dimension-six operator $Q_{H \square}$, and our expression (3.10) agrees with the results of the direct calculation of $\gamma_{H \square, H \square}$ presented in [3-5] — the found agreement constitutes a non- 
trivial cross-check of our computation. We add that the logarithmic corrections in (3.9) are scheme-independent, while the rational terms in $C_{H \square}^{(1)}$ depend on the choice of renormalisation scheme, including the specific treatment of $\phi$ tadpoles. Notice that the cancellation in (3.9) of logarithms that are not proportional to SM couplings is crucial to achieve the correct factorisation of short-distance and long-distance effects. In fact, in the SMEFT only the combination of SSM parameters that forms a Wilson coefficient has a non-trivial RG flow, together with the SM couplings $\lambda, g_{1}, g_{2}$ and $y_{f}$. Thus, the correct description of long-distance physics has to be formulated in terms of the SM couplings and the Wilson coefficient $C_{H \square}$ evaluated at the low-energy scale. Let us finally mention that (3.9) differs from the expression for $C_{H \square}^{(1)}$ given in both [26] and [29]. The disagreement has two sources. First, as shown in appendix A, the latter calculations miss certain heavy-loop contributions, and second, RG effects associated to the running of SSM parameters have not been explicitly included in the existing computations.

In the case of the operator $Q_{H}$, we have calculated the $H \rightarrow H, H H H \rightarrow H H H$, $H H^{\dagger} \rightarrow W_{\mu}^{a}$ and $H H \rightarrow H H W_{\mu}^{a} W_{\nu}^{b}$ scattering amplitudes to find the following expression for the one-loop correction to the Wilson coefficient $C_{H}$ :

$$
\begin{aligned}
C_{H}^{(1)}= & -\frac{\kappa^{3}}{12 M^{2}}-\frac{6 A^{2} \kappa \lambda_{\phi}+162 A^{2} \kappa \lambda-66 A^{2} \kappa^{2}-164 A^{2} \lambda^{2}+3 A \kappa^{2} \mu}{12 M^{4}} \\
& +\frac{87 A^{4} \kappa-6 A^{4} \lambda_{\phi}-72 A^{4} \lambda-60 A^{3} \kappa \mu+4 A^{3} \mu \lambda_{\phi}+78 A^{3} \lambda \mu+6 A^{2} \kappa \mu^{2}}{12 M^{6}} \\
& -\frac{8 A^{6}+21 A^{5} \mu-12 A^{4} \mu^{2}+2 A^{3} \mu^{3}}{12 M^{8}}-\frac{31 A^{2} \lambda g_{2}^{2}}{18 M^{4}} \\
& +\left(\gamma_{H, H \square} C_{H \square}^{(0)}+\gamma_{H, H} C_{H}^{(0)}\right) \ln \frac{\mu_{M}}{M} .
\end{aligned}
$$

The anomalous dimensions entering (3.11) read

$$
\begin{aligned}
\gamma_{H, H \square} & =-40 \lambda^{2}+\frac{20 \lambda g_{2}^{2}}{3}, \\
\gamma_{H, H} & =54 \lambda-\frac{9}{2}\left(g_{1}^{2}+3 g_{2}^{2}\right)+6 y_{2},
\end{aligned}
$$

while (3.6), (3.7) and (B.8) contain the explicit expressions for $C_{H \square}^{(0)}, C_{H}^{(0)}$ and $y_{2}$. All mass and coupling parameters that appear in (3.11) to (3.13) as well as in the tree-level expressions (3.6) and (3.7) are renormalised at the scale $M$.

Like in the case of (3.9), one observes that the logarithmic corrections in (3.11) involve only anomalous dimensions that depend on SM couplings, but not on SSM parameters. In fact, our expressions (3.12) and (3.13) for $\gamma_{H, H \square}$ and $\gamma_{H, H}$ agree with the results obtained in the articles [3-5]. The source of the difference between the first four terms in (3.11) and the rational terms of $C_{H}^{(1)}$ as quoted in $[26,29]$ is unraveled in appendix A. In addition, the existing calculations do not explicitly include effects stemming from the renormalisation of SSM parameters - cf. (B.2) to (B.5) - and therefore the logarithmic corrections given in (3.11) differ from the corresponding terms specified in [26, 29] as well.

In the case of the 15 dimension-six SMEFT operators in (3.4) that do not receive a tree-level Wilson coefficient, only heavy-light Feynman diagrams contribute to the one- 

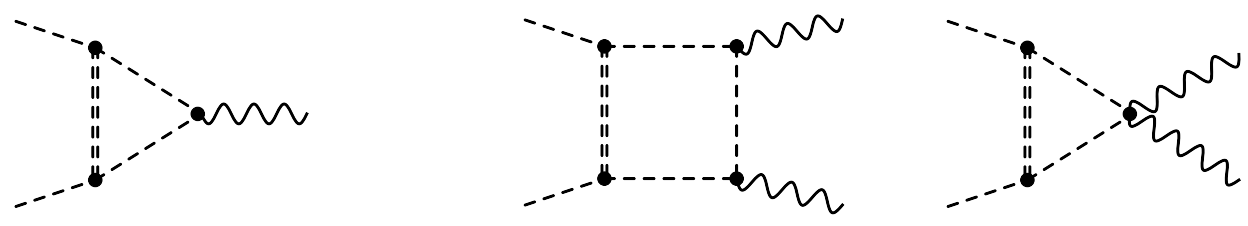

Figure 10. Examples of one-loop heavy-light scalar diagrams that need to be considered to extract the one-loop matching corrections of the bosonic dimension-six SMEFT operators in (3.4) that do not receive a tree-level Wilson coefficient. The line styles and their meanings resemble those of figure 5 .

loop matching. To extract the relevant one-loop corrections of the bosonic operators, we have calculated the off-shell amplitudes for $H H \rightarrow H H, H H^{\dagger} \rightarrow V_{\mu}$ and $H H^{\dagger} \rightarrow V_{\mu} V_{\nu}^{\prime}$ scattering with $V_{\mu}^{(\prime)}=B_{\mu}, W_{\mu}^{a}$. See figure 10 for the processes with external gauge bosons. We obtain

$$
\begin{aligned}
C_{H D}^{(1)} & =-\frac{31 A^{2} g_{1}^{2}}{18 M^{4}}+\gamma_{H D, H \square} C_{H \square}^{(0)} \ln \frac{\mu_{M}}{M}, \\
C_{H B}^{(1)} & =\frac{A^{2} g_{1}^{2}}{12 M^{4}}, \\
C_{H W}^{(1)} & =\frac{A^{2} g_{2}^{2}}{12 M^{4}}, \\
C_{H W B}^{(1)} & =\frac{A^{2} g_{1} g_{2}}{6 M^{4}} .
\end{aligned}
$$

Here

$$
\gamma_{H D, H \square}=\frac{20 g_{1}^{2}}{3},
$$

and the expression for the tree-level Wilson coefficient $C_{H \square}^{(0)}$ has already been given in (3.6). We emphasise that our results (3.14) to (3.17) agree with (A.20) to (A.23) of [29] and that the anomalous dimension (3.18) matches that calculated in [5]. Notice that in contrast to $C_{H D}^{(1)}$, the Wilson coefficients $C_{H B}^{(1)}, C_{H W}^{(1)}$ and $C_{H W B}^{(1)}$ do not receive logarithmic corrections. This feature is expected, because the tree-level operators $Q_{H \square}$ and $Q_{H}$ do not mix into $Q_{H B}, Q_{H W}$ and $Q_{H W B}$ at the one-loop level [3-5].

In order to determine the one-loop matching corrections of the fermionic dimensionsix SMEFT operators appearing in (3.4), we have computed the heavy-light scalar contributions to the $H H^{\dagger} \rightarrow \bar{f} f$ and $H H \rightarrow H \bar{f} f$ off-shell amplitudes with $f=q, u, d, \ell, e$. Examples of the corresponding diagrams are shown in figure 11. We find

$$
\begin{aligned}
C_{\psi H}^{(1)} & =-\frac{A^{2} y_{\psi}}{36 M^{4}}\left(27 \kappa-87 \lambda-\frac{9 A \mu}{M^{2}}+31 g_{2}^{2}-45 y_{\psi}^{\dagger} y_{\psi}\right)+\gamma_{\psi H, H \square} C_{H \square}^{(0)} \ln \frac{\mu_{M}}{M}, \\
C_{H u}^{(1)} & =-\frac{A^{2}}{216 M^{4}}\left(34 g_{1}^{2}-135 y_{u}^{\dagger} y_{u}\right)+\gamma_{H u, H \square} C_{H \square}^{(0)} \ln \frac{\mu_{M}}{M}, \\
C_{H d}^{(1)} & =\frac{A^{2}}{216 M^{4}}\left(17 g_{1}^{2}-135 y_{d}^{\dagger} y_{d}\right)+\gamma_{H d, H \square} C_{H \square}^{(0)} \ln \frac{\mu_{M}}{M},
\end{aligned}
$$



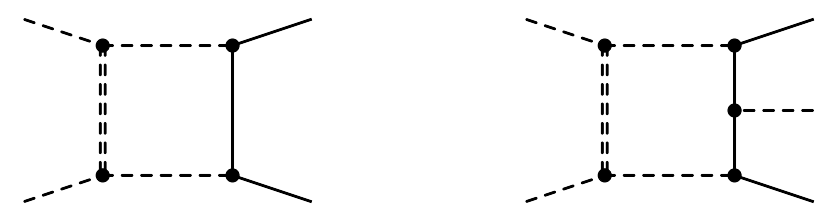

Figure 11. Examples of one-loop heavy-light scalar diagrams that need to be considered to extract the one-loop matching corrections of the fermionic dimension-six SMEFT operators in (3.4). The line styles and their meanings duplicate those of figure 3 .

$$
\begin{aligned}
C_{H e}^{(1)} & =\frac{A^{2}}{72 M^{4}}\left(17 g_{1}^{2}-45 y_{e}^{\dagger} y_{e}\right)+\gamma_{H e, H \square} C_{H \square}^{(0)} \ln \frac{\mu_{M}}{M}, \\
C_{H q^{(1)}}^{(1)} & =-\frac{A^{2}}{432 M^{4}}\left[17 g_{1}^{2}+135\left(y_{u} y_{u}^{\dagger}-y_{d} y_{d}^{\dagger}\right)\right]+\gamma_{H q^{(1)}, H \square} C_{H \square}^{(0)} \ln \frac{\mu_{M}}{M}, \\
C_{H q^{(3)}}^{(1)} & =-\frac{A^{2}}{144 M^{4}}\left[17 g_{2}^{2}-45\left(y_{u} y_{u}^{\dagger}+y_{d} y_{d}^{\dagger}\right)\right]+\gamma_{H q^{(3)}, H \square} C_{H \square}^{(0)} \ln \frac{\mu_{M}}{M}, \\
C_{H \ell^{(1)}}^{(1)} & =\frac{A^{2}}{144 M^{4}}\left(17 g_{1}^{2}+45 y_{e} y_{e}^{\dagger}\right)+\gamma_{H \ell^{(1)}, H \square} C_{H \square}^{(0)} \ln \frac{\mu_{M}}{M}, \\
C_{H \ell^{(3)}}^{(1)} & =-\frac{A^{2}}{144 M^{4}}\left(17 g_{2}^{2}-45 y_{e} y_{e}^{\dagger}\right)+\gamma_{H \ell^{(3)}, H \square} C_{H \square}^{(0)} \ln \frac{\mu_{M}}{M}, \\
C_{2 y}^{(1)} & =\frac{A^{2}}{6 M^{4}} .
\end{aligned}
$$

The one-loop anomalous dimensions appearing in the above expressions are

$$
\begin{aligned}
\gamma_{\psi H, H \square} & =-y_{\psi}\left(2 \lambda-\frac{10 g_{2}^{2}}{3}+6 y_{\psi}^{\dagger} y_{\psi}\right), \\
\gamma_{H u, H \square} & =\frac{2 g_{1}^{2}}{9}-y_{u}^{\dagger} y_{u}, \\
\gamma_{H d, H \square} & =-\frac{g_{1}^{2}}{9}+y_{d}^{\dagger} y_{d}, \\
\gamma_{H e, H \square} & =-\frac{g_{1}^{2}}{3}+y_{e}^{\dagger} y_{e}, \\
\gamma_{H q^{(1)}, H \square} & =\frac{g_{1}^{2}}{18}+\frac{1}{2}\left(y_{u} y_{u}^{\dagger}-y_{d} y_{d}^{\dagger}\right), \\
\gamma_{H q^{(3)}, H \square} & =\frac{g_{2}^{2}}{6}-\frac{1}{2}\left(y_{u} y_{u}^{\dagger}+y_{d} y_{d}^{\dagger}\right), \\
\gamma_{H \ell(1), H \square} & =-\frac{g_{1}^{2}}{6}-\frac{1}{2} y_{e} y_{e}^{\dagger}, \\
\gamma_{H \ell(3), H \square} & =\frac{g_{2}^{2}}{6}-\frac{1}{2} y_{e} y_{e}^{\dagger} .
\end{aligned}
$$

A sum over flavour indices is implicit in the above equations and the index $\psi$ in (3.19) and (3.28) can take the values $\psi=u, d, e$. Our results (3.19) to (3.27) for the one-loop matching corrections of the fermionic dimension-six SMEFT operators agree with (A.24) 
to (A.34) as given in [29]. In addition, the anomalous dimension expressions (3.28) to (3.35) fulfil the one-loop SMEFT RG equations collected in [3-5]. Finally, note that the operator $Q_{2 y}$ is a linear combination of several four-fermion operators in the Warsaw basis [54]. The Wilson coefficient $C_{2 y}$ does not receive a logarithmic correction, since the (purely bosonic) tree-level operators $Q_{H \square}$ and $Q_{H}$ obviously cannot mix into four-fermion operators at one loop.

\section{Acknowledgments}

We thank the authors of [26] and [29] for promptly confirming our results and for useful comments on the manuscript. We also thank Xiaochuan Lu for valuable feedback, and José Santiago for making us aware of the ongoing work [30] and helpful discussions. The Feynman diagrams shown in this paper have been drawn with JaxoDraw [55]. MR, EV and AW have been partially supported by the DFG Cluster of Excellence 2094 ORIGINS, the Collaborative Research Center SFB1258, the BMBF grant 05H18WOCA1 and thank the MIAPP for hospitality. MR is supported by the Studienstiftung des deutschen Volkes.

\section{A UOLEA results}

In this appendix we apply functional methods to perform the one-loop matching and point out some pieces which were missed in the previous calculation [26]. Once these missing parts are accounted for, the results obtained in the UOLEA framework agree with the expressions of our diagrammatic calculation described in section 3. We demonstrate this agreement explicitly for the contribution from heavy-particle loops to the one-loop matching corrections to the Wilson coefficients $C_{H \square}$ and $C_{H}$. Our discussion follows the general line of reasoning presented in the articles $[15,16,26]$ and we refer to the works [22-24] for CDE and UOLEA formulations including heavy-light loops.

Starting from the Lagrangian (2.3) one can obtain the low-energy effective action by performing the functional integral over the $\phi$ field. The part originating from heavy particle loops is given by

$$
\begin{aligned}
S_{\mathrm{eff}}[H] & \simeq S\left[H, \phi_{c}\right]+\frac{i}{2} \operatorname{Tr} \ln \left(-\left.\frac{\delta^{2} S}{\delta \phi^{2}}\right|_{\phi=\phi_{c}}\right) \\
& \simeq S\left[H, \phi_{c}\right]+\frac{i}{2} \operatorname{Tr} \ln \left(-P^{2}+M^{2}+U_{\phi}\right) .
\end{aligned}
$$

Here $\phi_{c}=\phi_{c}[H]$ is the solution of the classical equation of motion of $\phi$, i.e. $\delta S /\left.\delta \phi\right|_{\phi=\phi_{c}}=0$, $P_{\mu}=i \partial_{\mu}$ and $U_{\phi}$ in the case of (2.3) takes the form

$$
U_{\phi}=\left.\frac{\partial^{2} \mathcal{L}_{\phi}}{\partial \phi^{2}}\right|_{\phi=\phi_{c}}=\kappa|H|^{2}+\mu \phi_{c}+\frac{1}{2} \lambda_{\phi} \phi_{c}^{2}
$$


In order to find an expression for $\phi_{c}$ we perturbatively solve the equation of motion of $\phi$, which explicitly reads

$$
\left(\square+M^{2}+\kappa|H|^{2}\right) \phi=-A|H|^{2}-\frac{\mu}{2} \phi^{2}-\frac{\lambda_{\phi}}{6} \phi^{3} .
$$

Making the ansatz $\phi_{c}=\phi_{c}^{(0)}+\phi_{c}^{(1)}+\phi_{c}^{(2)}+\ldots$ with $\phi_{c}^{(k)}=O\left(\mu^{l} \lambda_{\phi}^{n}\right)$ and $k=l+n$ it is straightforward to obtain

$$
\begin{aligned}
& \phi_{c}^{(0)}=-\frac{1}{\square+M^{2}+\kappa|H|^{2}} A|H|^{2}, \\
& \phi_{c}^{(1)}=-\frac{1}{\square+M^{2}+\kappa|H|^{2}}\left(\frac{\mu}{2}\left(\phi_{c}^{(0)}\right)^{2}+\frac{\lambda_{\phi}}{6}\left(\phi_{c}^{(0)}\right)^{3}\right), \\
& \phi_{c}^{(2)}=-\frac{1}{\square+M^{2}+\kappa|H|^{2}}\left(\mu \phi_{c}^{(0)} \phi_{c}^{(1)}+\frac{\lambda_{\phi}}{2}\left(\phi_{c}^{(0)}\right)^{2} \phi_{c}^{(1)}\right) .
\end{aligned}
$$

Expanding $\phi_{c}$ up to four Higgs fields and two derivatives or six Higgs fields and no derivative, we then find the following expression

$$
\begin{aligned}
\phi_{c}= & -\frac{A}{M^{2}}|H|^{2}+\left(\frac{A \kappa}{M^{4}}-\frac{A^{2} \mu}{2 M^{6}}\right)|H|^{4}+\frac{A}{M^{4}} \square|H|^{2} \\
& -\left(\frac{A \kappa}{M^{6}}-\frac{A^{2} \mu}{M^{8}}\right)|H|^{2} \square|H|^{2}-\left(\frac{A \kappa^{2}}{M^{6}}-\frac{A^{3} \lambda_{\phi}+9 A^{2} \kappa \mu}{6 M^{8}}+\frac{A^{3} \mu^{2}}{2 M^{10}}\right)|H|^{6} .
\end{aligned}
$$

Comparing the above result for $\phi_{c}$ to (4.2) of [26] one observes that while the first three terms of (A.7) agree with the $|H|^{2},|H|^{4}$ and $\square|H|^{2}$ contributions given in the latter work, the $|H|^{2} \square|H|^{2}$ and $|H|^{6}$ contain additional pieces, all of which vanish in the limit $\mu \rightarrow 0$.

These additional terms affect the matching contributions from heavy loops to the oneloop Wilson coefficients $C_{H \square}^{(1)}$ and $C_{H}^{(1)}$, which consequently differ from the results presented in the work [26]. Considering the full solution of the classical equation of motion, we find that the heavy-loop contribution to $C_{H \square}^{(1)}$ is given by

$$
\begin{aligned}
C_{\left.H \square\right|_{\text {heavy }} ^{(1)}=} & -\left(\frac{A^{2} \lambda_{\phi}+A \kappa \mu}{2 M^{6}}-\frac{A^{2} \mu^{2}}{2 M^{8}}\right) \tilde{f}_{2} \\
& +\left(\frac{A \kappa \mu}{M^{4}}-\frac{A^{2} \mu^{2}}{M^{6}}\right) \tilde{f}_{4}+\left(\frac{\kappa^{2}}{2}-\frac{A \kappa \mu}{M^{2}}+\frac{A^{2} \mu^{2}}{2 M^{4}}\right) \tilde{f}_{7} \\
= & -\frac{\kappa^{2}}{24 M^{2}}-\frac{6 A^{2} \lambda_{\phi}+5 A \kappa \mu}{12 M^{4}}+\frac{11 A^{2} \mu^{2}}{24 M^{6}}-\frac{A^{2} \lambda_{\phi}}{M^{4}} \ln \frac{\mu_{M}}{M},
\end{aligned}
$$

where to obtain the final result we have inserted the expressions for the universal coefficients $\tilde{f}_{N}$ reported in appendix B of [26]. Notice that only the prefactor of $\tilde{f}_{2}$ in (A.8) differs from the result (4.10) presented in the article [26]. Diagrammatically the observed difference of $A^{2} \mu^{2}\left(1+2 \ln \mu_{M} / M\right) /\left(2 M^{6}\right)$ is due to propagator-type tadpole contributions see the last diagram in figure 2 - that have effectively been missed in the latter calculation. 
In the case of the heavy one-loop matching contributions to the Wilson coefficient of the operator $Q_{H}$ (cf. (3.2) and (3.4)), we instead obtain the following expression

$$
\begin{aligned}
\left.C_{H}^{(1)}\right|_{\text {heavy }}= & -\left(\frac{A^{2} \kappa \lambda_{\phi}+A \kappa^{2} \mu}{2 M^{6}}-\frac{4 A^{3} \mu \lambda_{\phi}+9 A^{2} \kappa \mu^{2}}{12 M^{8}}+\frac{A^{3} \mu^{3}}{4 M^{10}}\right) \tilde{f}_{2} \\
& +\left(\frac{A^{2} \kappa \lambda_{\phi}+2 A \kappa^{2} \mu}{2 M^{4}}-\frac{A^{3} \mu \lambda_{\phi}+3 A^{2} \kappa \mu^{2}}{2 M^{6}}+\frac{A^{3} \mu^{3}}{2 M^{8}}\right) \tilde{f}_{4} \\
& +\left(\frac{\kappa^{3}}{2}-\frac{3 A \kappa^{2} \mu}{2 M^{2}}+\frac{3 A^{2} \kappa \mu^{2}}{2 M^{4}}-\frac{A^{3} \mu^{3}}{2 M^{6}}\right) \tilde{f}_{8} \\
= & -\frac{\kappa^{3}}{12 M^{2}}-\frac{2 A^{2} \kappa \lambda_{\phi}+A \kappa^{2} \mu}{4 M^{4}}+\frac{2 A^{3} \mu \lambda_{\phi}+3 A^{2} \kappa \mu^{2}}{6 M^{6}}-\frac{A^{3} \mu^{3}}{6 M^{8}} \\
& -\left(\frac{A^{2} \kappa \lambda_{\phi}}{2 M^{4}}-\frac{A^{3} \mu \lambda_{\phi}}{6 M^{6}}\right) \ln \frac{\mu_{M}}{M} .
\end{aligned}
$$

Apart from the prefactor of $\tilde{f}_{2}$, the latter result agrees with (4.9) of [26]. The resulting difference of $A^{2} \mu^{2}\left(A \mu-2 M^{2} \kappa\right)\left(1+2 \ln \mu_{M} / M\right) /\left(4 M^{8}\right)$ can again be traced back to propagator-type tadpole contributions that have not been correctly included in the latter article. Our formula (A.9) is in accord with the preliminary results presented in the talk [30], where small discrepancies with the formula for $C_{H}^{(1)}$ given in [26] were already observed.

The heavy-light contributions to the one-loop matching corrections to the dimensionsix SMEFT operators $Q_{H \square}$ and $Q_{H}$ are not affected by the additional terms in (A.7). In fact, the operators generated by heavy-light loops that are proportional to $\phi_{c}$ appear with at least two additional Higgs fields compared to $Q_{H \square}$ and $Q_{H}$. As a result the missing terms only affect the one-loop matching of operators with a mass dimension of eight or higher. However, when trying to reproduce the results in [26] we discovered an unrelated typo in the heavy-light contribution to $C_{H}^{(1)}$ that appears in the prefactor of $\tilde{f}_{4 A}$ in (4.8) of that work. We find that the actual contribution of the universal coefficient $\tilde{f}_{4 A}$ to the Wilson coefficient $C_{H}^{(1)}$ should read

$$
\left.C_{H}^{(1)}\right|_{\tilde{f}_{4 A}}=\frac{3 A^{2} \kappa^{2}}{M^{4}}-\frac{A^{3} \kappa \mu}{M^{6}} .
$$

Once the additional terms in (A.7) and the correction (A.10) are taken into account we recover the diagrammatic results for $C_{H \square}^{(1)}$ and $C_{H}^{(1)}$ originating from heavy and heavy-light pure scalar loops, meaning that our UOLEA calculation reproduced the terms in the first two lines of (3.9) as well as the terms in the first three lines of (3.11). Note that the above mistakes and typos are also present in (3.1) and (3.2) of [29], which employed the UOLEA master formulas of [26] to obtain the aforementioned results.

\section{B RG evolution of SSM parameters}

We define the one-loop anomalous dimensions $\gamma_{x}$ that enter the RG evolution of the parameters $x$ by

$$
\frac{d x}{d \ln \mu_{R}}=\frac{\gamma_{x}}{(4 \pi)^{2}}
$$


Renormalising all UV poles including those arising from $\phi$ tadpoles in the $\overline{\mathrm{MS}}$ scheme, the anomalous dimensions of the parameters $M^{2}, A, \kappa, \mu, \lambda_{\phi}$ and $\lambda_{h}$ read

$$
\begin{aligned}
\gamma_{M^{2}} & =M^{2} \lambda_{\phi}+4 A^{2}, \\
\gamma_{A} & =A\left(4 \kappa+6 \lambda_{h}-\frac{3}{2}\left(g_{1}^{2}+3 g_{2}^{2}\right)+2 y_{2}\right), \\
\gamma_{\kappa} & =\kappa\left(4 \kappa+\lambda_{\phi}+6 \lambda_{h}-\frac{3}{2}\left(g_{1}^{2}+3 g_{2}^{2}\right)+2 y_{2}\right), \\
\gamma_{\mu} & =2 \mu \lambda_{\phi}+12 A \kappa \\
\gamma_{\lambda_{\phi}} & =12 \kappa^{2}+3 \lambda_{\phi}^{2}, \\
\gamma_{\lambda_{h}} & =\kappa^{2}+12 \lambda_{h}^{2}-3 \lambda_{h}\left(g_{1}^{2}+3 g_{2}^{2}\right)+\frac{3}{4}\left(g_{1}^{4}+2 g_{1}^{2} g_{2}^{2}+3 g_{2}^{4}\right)+4 \lambda_{h} y_{2}-4 y_{4},
\end{aligned}
$$

with

$$
\begin{aligned}
& y_{2}=\operatorname{Tr}\left(3 y_{u}^{\dagger} y_{u}+3 y_{d}^{\dagger} y_{d}+y_{e}^{\dagger} y_{e}\right) \\
& y_{4}=\operatorname{Tr}\left(3 y_{u}^{\dagger} y_{u} y_{u}^{\dagger} y_{u}+3 y_{d}^{\dagger} y_{d} y_{d}^{\dagger} y_{d}+y_{e}^{\dagger} y_{e} y_{e}^{\dagger} y_{e}\right) .
\end{aligned}
$$

Examples of Feynman graphs that contribute to the anomalous dimensions $\gamma_{M^{2}}, \gamma_{A}, \gamma_{\kappa}$ and $\gamma_{\mu}$ are shown on the right of figure 3 as well as in figure 6 to figure 9. We add that the results for $\gamma_{\lambda_{\phi}}$ and $\gamma_{\lambda_{h}}$ are not needed in the context of this work, but we provide them for completeness.

In section 3.2 of this article we have presented our final results (3.9) and (3.11) for the one-loop matching corrections $C_{H \square}^{(1)}$ and $C_{H}^{(1)}$. In both cases we have observed that the logarithmic corrections to the Wilson coefficients involve only anomalous dimensions that depend only on SM couplings but not on SSM parameters. Below we explicitly show how this feature arises. The given formulae should also facilitate a comparison to the existing computations $[26,29]$ as well as to the preliminary results presented in the talk [30].

In order to derive the logarithmic terms that arise from the renormalisation of the SSM parameters, we first notice that if the tree-level Wilson coefficients $C_{k}^{(0)}$ are expressed through the SSM parameters $x$ one has

$$
\frac{d C_{k}^{(0)}}{d \ln \mu_{R}}=\sum_{x} \frac{\partial C_{k}^{(0)}}{\partial x} \frac{d x}{d \ln \mu_{R}}=\frac{1}{(4 \pi)^{2}} \sum_{x} \frac{\partial C_{k}^{(0)}}{\partial x} \gamma_{x},
$$

where the sum over $x$ includes $M^{2}, A, \kappa, \mu, \lambda_{\phi}$ and $\lambda_{h}$, and in the last step we have used the definition (B.1). Integrating (B.10) from $\mu_{M}$ to $M$ then gives rise to the logarithmic corrections that are associated to the renormalisation of the SSM parameters.

Applying the master formula (B.10) to the case of the Wilson coefficient $C_{H \square}^{(1)}$, we find the following logarithmic terms

$$
\begin{aligned}
\left.C_{H \square}^{(1)}\right|_{\ln \frac{\mu_{M}}{M}} & =\frac{4 A^{2} \kappa-A^{2} \lambda_{\phi}}{M^{4}}+\frac{2 A^{4}}{M^{6}}-\frac{5 A^{2}\left(g_{1}^{2}+3 g_{2}^{2}\right)}{6 M^{4}}+\frac{A^{2} \gamma_{M^{2}}}{M^{6}}-\frac{A \gamma_{A}}{M^{4}} \\
& =\left(12 \lambda-\frac{4}{3}\left(g_{1}^{2}+3 g_{2}^{2}\right)+4 y_{2}\right)\left(-\frac{A^{2}}{2 M^{4}}\right) .
\end{aligned}
$$


The first three terms in the first line of (B.11) result from the heavy and heavy-light loop diagrams shown in figure 2 to figure 5. The terms proportional to $\gamma_{M^{2}}$ and $\gamma_{A}$ instead arise from the renormalisation of the parameters $M^{2}$ and $A$ that enter the treelevel Wilson coefficient (3.6). In the second line of (B.11) we can manifestly see that there is a cancellation of logarithmic terms involving the combinations of SSM parameters that do not form a SMEFT Wilson coefficient, yielding the logarithmic correction quoted in (3.9) as final result.

In the case of the Wilson coefficient $C_{H}^{(1)}$ using (B.10) instead leads to

$$
\begin{aligned}
\left.C_{H}^{(1)}\right|_{\ln \frac{\mu_{M}}{M}=} & -\frac{A^{2} \kappa \lambda_{\phi}+36 A^{2} \kappa \lambda-12 A^{2} \kappa^{2}-40 A^{2} \lambda^{2}}{2 M^{4}} \\
& +\frac{18 A^{4} \kappa-12 A^{3} \kappa \mu+A^{3} \mu \lambda_{\phi}+36 A^{3} \lambda \mu}{6 M^{6}}-\frac{A^{5} \mu}{M^{8}}-\frac{10 A^{2} \lambda g_{2}^{2}}{3 M^{4}} \\
& +\left(\frac{A^{2} \kappa}{M^{6}}-\frac{A^{3} \mu}{2 M^{8}}\right) \gamma_{M^{2}}-\left(\frac{A \kappa}{M^{4}}-\frac{A^{2} \mu}{2 M^{6}}\right) \gamma_{A}-\frac{A^{2} \gamma_{\kappa}}{2 M^{4}}+\frac{A^{3} \gamma_{\mu}}{6 M^{6}} \\
= & \left(-40 \lambda^{2}+\frac{20 \lambda g_{2}^{2}}{3}\right)\left(-\frac{A^{2}}{2 M^{4}}\right) \\
& +\left(54 \lambda-\frac{9}{2}\left(g_{1}^{2}+3 g_{2}^{2}\right)+6 y_{2}\right)\left(\frac{A^{3} \mu}{6 M^{6}}-\frac{A^{2} \kappa}{2 M^{4}}\right) .
\end{aligned}
$$

The first two lines of the above expression correspond to the contributions from heavy and heavy-light graphs, while the terms proportional to the anomalous dimensions $\gamma_{M^{2}}, \gamma_{A}$, $\gamma_{\kappa}$ and $\gamma_{\mu}$ are the counterterm contributions that are associated to the renormalisation of the relevant SSM parameters appearing in the tree-level Wilson coefficient $C_{H}^{(0)}$. Notice that the final result in (B.12) agrees with the logarithmic correction that we have obtained in (3.11), and that these terms have the correct form to allow for a resummation of large logarithms using the RG equations of the dimension-six SMEFT operators $Q_{H \square}$ and $Q_{H}$ derived in $[3-5]$.

Open Access. This article is distributed under the terms of the Creative Commons Attribution License (CC-BY 4.0), which permits any use, distribution and reproduction in any medium, provided the original author(s) and source are credited.

\section{References}

[1] B. Grzadkowski, M. Iskrzynski, M. Misiak and J. Rosiek, Dimension-Six Terms in the Standard Model Lagrangian, JHEP 10 (2010) 085 [arXiv: 1008.4884] [INSPIRE].

[2] W. Buchmüller and D. Wyler, Effective Lagrangian Analysis of New Interactions and Flavor Conservation, Nucl. Phys. B 268 (1986) 621 [INSPIRE].

[3] E.E. Jenkins, A.V. Manohar and M. Trott, Renormalization Group Evolution of the Standard Model Dimension Six Operators I: Formalism and lambda Dependence, JHEP 10 (2013) 087 [arXiv: 1308.2627] [INSPIRE]. 
[4] E.E. Jenkins, A.V. Manohar and M. Trott, Renormalization Group Evolution of the Standard Model Dimension Six Operators II: Yukawa Dependence, JHEP 01 (2014) 035 [arXiv: 1310.4838] [INSPIRE].

[5] R. Alonso, E.E. Jenkins, A.V. Manohar and M. Trott, Renormalization Group Evolution of the Standard Model Dimension Six Operators III: Gauge Coupling Dependence and Phenomenology, JHEP 04 (2014) 159 [arXiv:1312.2014] [INSPIRE].

[6] R. Alonso, H.-M. Chang, E.E. Jenkins, A.V. Manohar and B. Shotwell, Renormalization group evolution of dimension-six baryon number violating operators, Phys. Lett. B 734 (2014) 302 [arXiv: 1405. 0486] [INSPIRE].

[7] C. Grojean, E.E. Jenkins, A.V. Manohar and M. Trott, Renormalization Group Scaling of Higgs Operators and $\Gamma(h \rightarrow \gamma \gamma)$, JHEP 04 (2013) 016 [arXiv: 1301.2588] [INSPIRE].

[8] J. Elias-Miró, J.R. Espinosa, E. Masso and A. Pomarol, Higgs windows to new physics through $d=6$ operators: constraints and one-loop anomalous dimensions, JHEP 11 (2013) 066 [arXiv: 1308.1879] [INSPIRE].

[9] C. Zhang, Effective field theory approach to top-quark decay at next-to-leading order in QCD, Phys. Rev. D 90 (2014) 014008 [arXiv: 1404.1264] [INSPIRE].

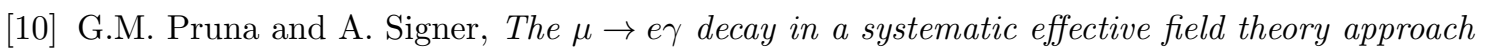
with dimension 6 operators, JHEP 10 (2014) 014 [arXiv:1408.3565] [INSPIRE].

[11] J. Brod, A. Greljo, E. Stamou and P. Uttayarat, Probing anomalous $t \bar{t} Z$ interactions with rare meson decays, JHEP 02 (2015) 141 [arXiv: 1408.0792] [INSPIRE].

[12] C. Cheung and C.-H. Shen, Nonrenormalization Theorems without Supersymmetry, Phys. Rev. Lett. 115 (2015) 071601 [arXiv: 1505.01844] [INSPIRE].

[13] M. Gorbahn and U. Haisch, Indirect probes of the trilinear Higgs coupling: $g g \rightarrow h$ and $h \rightarrow \gamma \gamma$, JHEP 10 (2016) 094 [arXiv: 1607. 03773] [INSPIRE].

[14] J. de Blas, J.C. Criado, M. Pérez-Victoria and J. Santiago, Effective description of general extensions of the Standard Model: the complete tree-level dictionary, JHEP 03 (2018) 109 [arXiv: 1711.10391] [INSPIRE].

[15] A. Drozd, J. Ellis, J. Quevillon and T. You, The Universal One-Loop Effective Action, JHEP 03 (2016) 180 [arXiv: 1512.03003] [INSPIRE].

[16] B. Henning, X. Lu and H. Murayama, How to use the Standard Model effective field theory, JHEP 01 (2016) 023 [arXiv: 1412.1837] [INSPIRE].

[17] M.K. Gaillard, The Effective One Loop Lagrangian With Derivative Couplings, Nucl. Phys. B 268 (1986) 669 [INSPIRE].

[18] L.-H. Chan, Derivative Expansion for the One Loop Effective Actions With Internal Symmetry, Phys. Rev. Lett. 57 (1986) 1199 [INSPIRE].

[19] O. Cheyette, Effective Action for the Standard Model With Large Higgs Mass, Nucl. Phys. B 297 (1988) 183 [INSPIRE].

[20] F. del Aguila, Z. Kunszt and J. Santiago, One-loop effective lagrangians after matching, Eur. Phys. J. C 76 (2016) 244 [arXiv: 1602.00126] [inSPIRE].

[21] M. Boggia, R. Gomez-Ambrosio and G. Passarino, Low energy behaviour of standard model extensions, JHEP 05 (2016) 162 [arXiv: 1603.03660] [INSPIRE]. 
[22] B. Henning, X. Lu and H. Murayama, One-loop Matching and Running with Covariant Derivative Expansion, JHEP 01 (2018) 123 [arXiv:1604.01019] [INSPIRE].

[23] S.A.R. Ellis, J. Quevillon, T. You and Z. Zhang, Mixed heavy-light matching in the Universal One-Loop Effective Action, Phys. Lett. B 762 (2016) 166 [arXiv:1604.02445] [INSPIRE].

[24] J. Fuentes-Martin, J. Portoles and P. Ruiz-Femenia, Integrating out heavy particles with functional methods: a simplified framework, JHEP 09 (2016) 156 [arXiv:1607.02142] [INSPIRE].

[25] Z. Zhang, Covariant diagrams for one-loop matching, JHEP 05 (2017) 152 [arXiv: 1610.00710] [INSPIRE].

[26] S.A.R. Ellis, J. Quevillon, T. You and Z. Zhang, Extending the Universal One-Loop Effective Action: Heavy-Light Coefficients, JHEP 08 (2017) 054 [arXiv: 1706. 07765] [INSPIRE].

[27] M. Krämer, B. Summ and A. Voigt, Completing the scalar and fermionic Universal One-Loop Effective Action, JHEP 01 (2020) 079 [arXiv:1908.04798] [INSPIRE].

[28] T. Cohen, M. Freytsis and X. Lu, Functional Methods for Heavy Quark Effective Theory, arXiv:1912.08814 [INSPIRE].

[29] M. Jiang, N. Craig, Y.-Y. Li and D. Sutherland, Complete One-Loop Matching for a Singlet Scalar in the Standard Model EFT, JHEP 02 (2019) 031 [arXiv:1811.08878] [INSPIRE].

[30] C. Anastasiou, A. Carmona, A. Lazopoulos and J. Santiago, Match Maker, talk given at SMEFT-TOOLS 2019, Durham U.K. (2019), https://indico.cern.ch/event/787665/contributions/3374418/attachments/1861555/3059604/ JSantiagoSMEFT-TOOLS19.pdf.

[31] U. Haisch, M. Ruhdorfer, E. Salvioni, E. Venturini and A. Weiler, in preparation.

[32] M. Frigerio, A. Pomarol, F. Riva and A. Urbano, Composite Scalar Dark Matter, JHEP 07 (2012) 015 [arXiv:1204.2808] [INSPIRE].

[33] R. Balkin, M. Ruhdorfer, E. Salvioni and A. Weiler, Dark matter shifts away from direct detection, JCAP 11 (2018) 050 [arXiv:1809.09106] [INSPIRE].

[34] V. Barger, P. Langacker, M. McCaskey, M. Ramsey-Musolf and G. Shaughnessy, Complex Singlet Extension of the Standard Model, Phys. Rev. D 79 (2009) 015018 [arXiv:0811.0393] [INSPIRE].

[35] C. Gross, O. Lebedev and T. Toma, Cancellation Mechanism for Dark-Matter-Nucleon Interaction, Phys. Rev. Lett. 119 (2017) 191801 [arXiv:1708.02253] [INSPIRE].

[36] M. Ruhdorfer, E. Salvioni and A. Weiler, A Global View of the Off-Shell Higgs Portal, SciPost Phys. 8 (2020) 027 [arXiv: 1910.04170] [InSPIRE].

[37] G.F. Giudice, C. Grojean, A. Pomarol and R. Rattazzi, The Strongly-Interacting Light Higgs, JHEP 06 (2007) 045 [hep-ph/0703164] [INSPIRE].

[38] C. Bobeth, M. Misiak and J. Urban, Photonic penguins at two loops and $m_{t}$ dependence of $B R\left[B \rightarrow X_{s} l^{+} l^{-}\right]$, Nucl. Phys. B 574 (2000) 291 [hep-ph/9910220] [INSPIRE].

[39] P. Gambino and U. Haisch, Complete electroweak matching for radiative B decays, JHEP 10 (2001) 020 [hep-ph/0109058] [INSPIRE].

[40] T. Hahn, Generating Feynman diagrams and amplitudes with FeynArts 3, Comput. Phys. Commun. 140 (2001) 418 [hep-ph/0012260] [INSPIRE]. 
[41] A. Alloul, N.D. Christensen, C. Degrande, C. Duhr and B. Fuks, FeynRules 2.0 - A complete toolbox for tree-level phenomenology, Comput. Phys. Commun. 185 (2014) 2250 [arXiv:1310.1921] [INSPIRE].

[42] T. Hahn and M. Pérez-Victoria, Automatized one loop calculations in four-dimensions and D-dimensions, Comput. Phys. Commun. 118 (1999) 153 [hep-ph/9807565] [InSPIRE].

[43] H.H. Patel, Package-X: A Mathematica package for the analytic calculation of one-loop integrals, Comput. Phys. Commun. 197 (2015) 276 [arXiv:1503.01469] [INSPIRE].

[44] F. Jegerlehner, M. Yu. Kalmykov and O. Veretin, MS versus pole masses of gauge bosons: Electroweak bosonic two loop corrections, Nucl. Phys. B 641 (2002) 285 [hep-ph/0105304] [INSPIRE].

[45] F. Jegerlehner, M. Yu. Kalmykov and O. Veretin, MS-bar versus pole masses of gauge bosons. 2. Two loop electroweak fermion corrections, Nucl. Phys. B 658 (2003) 49 [hep-ph/0212319] [INSPIRE].

[46] A. Denner, Techniques for calculation of electroweak radiative corrections at the one loop level and results for W physics at LEP-200, Fortsch. Phys. 41 (1993) 307 [arXiv:0709.1075] [INSPIRE].

[47] J. Fleischer and F. Jegerlehner, Radiative Corrections to Higgs Decays in the Extended Weinberg-Salam Model, Phys. Rev. D 23 (1981) 2001 [INSPIRE].

[48] A. Denner, L. Jenniches, J.-N. Lang and C. Sturm, Gauge-independent $\overline{M S}$ renormalization in the 2HDM, JHEP 09 (2016) 115 [arXiv:1607.07352] [INSPIRE].

[49] J.M. Cullen, B.D. Pecjak and D.J. Scott, NLO corrections to $h \rightarrow b \bar{b}$ decay in SMEFT, JHEP 08 (2019) 173 [arXiv: 1904.06358] [INSPIRE].

[50] A.J. Buras, Weak Hamiltonian, CP-violation and rare decays, in Probing the standard model of particle interactions. Proceedings of Summer School in Theoretical Physics, NATO

Advanced Study Institute, 68th session, Les Houches France (1997), pg. 281 [hep-ph/9806471] [INSPIRE].

[51] P. Gambino, A. Kwiatkowski and N. Pott, Electroweak effects in the $B^{0}-B^{0}$ mixing, Nucl. Phys. B 544 (1999) 532 [hep-ph/9810400] [INSPIRE].

[52] A.J. Buras, P. Gambino and U.A. Haisch, Electroweak penguin contributions to nonleptonic $\Delta F=1$ decays at NNLO, Nucl. Phys. B 570 (2000) 117 [hep-ph/9911250] [InSPIRE].

[53] T. Cohen, As Scales Become Separated: Lectures on Effective Field Theory, PoS (TASI2018) 011 [arXiv: 1903.03622] [INSPIRE].

[54] J.D. Wells and Z. Zhang, Effective theories of universal theories, JHEP 01 (2016) 123 [arXiv: 1510.08462] [INSPIRE].

[55] D. Binosi, J. Collins, C. Kaufhold and L. Theussl, JaxoDraw: A Graphical user interface for drawing Feynman diagrams. Version 2.0 release notes, Comput. Phys. Commun. 180 (2009) 1709 [arXiv:0811.4113] [INSPIRE]. 